Research Article

\title{
FOXP in Tetrapoda: Intrinsically Disordered Regions, Short Linear Motifs and their evolutionary significance
}

\author{
Lucas Henriques Viscardi ${ }^{1}$, Luciana Tovo-Rodrigues ${ }^{2}$, Pamela Paré ${ }^{1}$, Nelson Jurandi Rosa Fagundes ${ }^{1}$, \\ Francisco Mauro Salzano ${ }^{1}$, Vanessa Rodrigues Paixão-Côrtes ${ }^{3}$, Claiton Henrique Dotto Bau ${ }^{1}$ and Maria \\ Cátira Bortolini ${ }^{1}$ \\ ${ }^{1}$ Programa de Pós-Graduação em Genética e Biologia Molecular, Departamento de Genética, Universidade \\ Federal do Rio Grande do Sul, Porto Alegre, RS, Brazil. \\ ${ }^{2}$ Programa de Pós-Graduação em Epidemiologia, Universidade Federal de Pelotas, Pelotas, RS, Brazil. \\ ${ }^{3}$ Programa de Pós-Graduação em Genética e Biodiversidade, Instituto de Biologia, Universidade Federal \\ da Bahia, Salvador, BA, Brazil.
}

\begin{abstract}
The FOXP subfamily is probably the most extensively characterized subfamily of the forkhead superfamily, playing important roles in development and homeostasis in vertebrates. Intrinsically disorder protein regions (IDRs) are protein segments that exhibit multiple physical interactions and play critical roles in various biological processes, including regulation and signaling. IDRs in proteins may play an important role in the evolvability of genetic systems. In this study, we analyzed 77 orthologous FOXP genes/proteins from Tetrapoda, regarding protein disorder content and evolutionary rate. We also predicted the number and type of short linear motifs (SLIMs) in the IDRs. Similar levels of protein disorder (approximately 70\%) were found for FOXP1, FOXP2, and FOXP4. However, for FOXP3, which is shorter in length and has a more specific function, the disordered content was lower (30\%). Mammals showed higher protein disorders for FOXP1 and FOXP4 than non-mammals. Specific analyses related to linear motifs in the four genes showed also a clear differentiation between FOXPs in mammals and non-mammals. We predicted for the first time the role of IDRs and SLIMs in the FOXP gene family associated with possible adaptive novelties within Tetrapoda. For instance, we found gain and loss of important phosphorylation sites in the Homo sapiens FOXP2 IDR regions, with possible implication for the evolution of human speech.
\end{abstract}

Keywords: FOXP2, Intrinsically disordered protein regions, forkhead superfamily, short linear motif, molecular evolution.

Received: May 25, 2016; Accepted: October 03, 2016.

\section{Introduction}

Members of the Forkhead box (FOX) gene superfamily have been widely associated with organismal development and are identified by their evolutionary conserved forkhead DNA-binding domain (Lam et al., 2013; Morris and Fanucchi, 2016). The FOXP subfamily is probably the most extensively characterized subfamily of the forkhead superfamily. The four FOXP genes (FOXP1, FOXP2 $F O X P 3$, and FOXP4) emerged by duplication events during the origin of vertebrates (Santos et al., 2011; Song et al., 2016). Since the duplication events, paralogues FOXP1, FOXP2, and FOXP4 have played an important role in brain, lung, heart, and jaw development in vertebrates, while FOXP3 has been associated with the development and homeostasis of the immune system, since it is described

Send correspondence to Maria Cátira Bortolini. Departamento de Genética, Instituto de Biociências, Universidade Federal do Rio Grande do Sul, Campus do Vale, Caixa Postal 15053, 91501-970 Porto Alegre, RS, Brazil. E-mail: maria.bortolini@ufrgs.br. as a master-regulator of $\mathrm{CD} 4{ }^{+}$and $\mathrm{CD} 25^{+} \mathrm{T}$-cells (Coffer and Burgering, 2004; Akbar et al., 2007; Takahashi et al., 2008; Benayoun et al., 2011; Andersen et al., 2012; Lam et al., 2013; Cesario et al., 2016).

Undoubtedly, the most widely known member of the FOXP subfamily is FOXP2, as it has attracted the attention of the scientific community and the general media because of its role in the evolution of speech and vocalization in mammals (Zhang et al., 2002; Li et al., 2007), especially because mutations in this gene promote severe impairment of articulation and grammar in humans (Enard et al., 2002; Schön et al., 2006; Enard, 2011; Bowers and Konopka, 2012). FOXP2 is expressed primarily in the brain, where it plays an important role in synapse formation and cell adhesion, as well as in the specification and differentiation of the lung epithelium and gastrointestinal and cardiovascular tissues (Song et al., 2013).

Evolutionary studies have been successively improved by incorporating new methodological approaches. 
Analysis of intrinsically disordered regions (IDRs), which is routinely used in medical and structural biology studies, can also be applied in evolutionary studies because of the possible role of IDRs in the evolvability (evolutionary capacity; Pigliucci, 2008; Xue et al., 2010b, 2013) of genetic systems (Neduva and Russell, 2005). IDRs are protein segments rich in hydrophilic, polar, and charged amino acids (glutamine, serine, glutamic acid, arginine, and lysine), as well as glycine, proline, and alanine (Iakoucheva et al., 2004; Liu et al., 2006). IDRs are prevalent in proteins that exhibit multiple physical interactions and play critical roles in various biological processes, including regulation and signaling (Dunker et al., 2000; Nguyen Ba et al., 2012; Forman-Kay and Mittag, 2013). The conformational flexibility of IDRs facilitates exposure of specific residues for modification and binding to other proteins and molecules (Huang and Sarai, 2012; Liu and Huang, 2014). Thus, intrinsically disordered proteins (IDPs) are characterized by a high IDR content and the absence of stable well-folded three-dimensional structures in solutions (Forman-Kay and Mittag, 2013).

Short linear motifs (SLIMs) are short stretches in protein sequences that mediate protein-protein interactions. SLIMs are typically 2-10 amino acids long; however, only two or three amino acids are essential for interaction with other molecules. SLIMs are common elements in IDRs, and they probably play a significant role in the functioning of these disordered regions (Wagner and Lynch, 2008; Huang and Sarai, 2012; Nguyen Ba et al., 2012; FormanKay and Mittag, 2013; Liu and Huang, 2014). The presence of a great number of these motifs in such regions probably confers functional flexibility to this class of proteins (Gould et al., 2010; Disfani et al., 2012; Dinkel et al., 2012, 2014). Furthermore, SLIMs are particularly evolvable because they are poorly conserved between lineages and can appear and disappear through small changes (Wagner and Lynch, 2008). Therefore, changes in SLIMs significantly impact complex regulatory networks (Neduva and Russell, 2005). Thus, analysis of these changes enables the assessment of their importance in the evolutionary trajectory of animals.

In addition to the forkhead, leucine-zipper, and zincfinger domains, other molecular elements such as IDRs may play crucial roles in the function of FOXP proteins. However, these structures have not been studied extensively. Thus, the present investigation aims to ask how FOXPs structural forms changed throughout Tetrapoda evolution regarding linear motifs composition and disordered content. Furthermore, as FOXP3 is known to be the only gene among the FOXP family playing a role in the immune system, we investigated if a higher evolutionary rate would be observed when compared with other FOXPs, and if such a rate could be related with higher disordered content.

\section{Material and Methods}

Seventy-seven orthologues FOXP genes/proteins from tetrapods (Table S1) were considered in the present study. FOXP nucleotide sequences were retrieved from the NCBI database using BLASTN with 20,000 Max target sequences. We also used the Ensembl genome database (http://ensembl.org/) for sequence retrieval. The Neanderthal exome (Castellano et al., 2014; http://cdna.eva.mpg.de/) was consulted to verify possible specific changes within the genus Homo. However, one protein-coding gene may codify more than one isoform. The presence of many isoforms in the FOX genes, caused by alternative splicing, was handled conservatively by choosing only isoforms that clearly resemble the canonical form identified in humans by using UniProt (http://www.uniprot.org/). Incomplete sequences were removed from the analysis. Subsequently, the sequences were aligned using the MAFFT algorithms (standard pattern) implemented in the Guidance web server (http://guidance.tau.ac.il/). The alignments are available in the Supplementary Material. Phylogenetic trees were drawn using FigTree1.4.2. (http://tree.bio.ed.ac.uk/software/figtree/) according to the literature (Meredith et al., 2011; Perelman et al., 2011; Song et al., 2012).

Importantly, while both FOXP2 and FOXP4 passed through a standard NsSites test site analysis, for FOXP3 and FOXP1 we had to employ distinct data tests. Because of the absence of several base pairs in Xenopus laevis FOXP1, we excluded this species. For FOXP3, just the mammalian sequences were used because reptilian and amphibian FOXP3 are shorter and very different, while in birds, FOXP3 is completely absent (Andersen et al., 2012). In addition, we removed from the analysis a residual $\mathrm{N}$-terminal part of FOXP3 present only in the mammals Nomascus leucogenys Papio anubis, Chlorocebus sabaeus, Callithrix jacchus, Cricetulus griseus, Panthera tigris, Myotis brandtii, Pteropus alecto, Chrysochloris asiatica, and Dasypus novemcinctus, as they do not align or resemble other orthologous and known isoforms.

We predicted disordered regions by using the PONDR-FIT metapredictor (Xue et al., 2010a). Additionally, the MobiDB server (Potenza et al., 2014) was consulted to check consensus predictions for their disorder content, as provided by a variety of disorder predictors. SLIMs were predicted using the ELM webserver (Dinkel et al., 2012, 2014) considering only the cell nucleus as the cell compartment for biochemical interaction context of FOXP proteins. Given that the linear motifs predicted by ELM can present a high rate of false positives, we considered only ELM in IDR regions and validated such predictions by analyzing the literature on the interactions between linear motifs and their ligands with other transcription factors. Therefore, we considered only linear motifs with confirmed experimental data and/or certainty for ELM reliability annotation. All information regarding the linear motifs 
was retrieved from the ELM server and from the literature. The ELM server classifies SLIMs into the following four types: protease cleavage sites, protein motif interaction/binding sites, posttranslational modification sites, and subcellular targeting signals (Dinkel et al., 2012). Linear motifs present in the forkhead, leucine-zipper, and zinc-finger domains were not considered because they can represent false positives. Statistical tests comparing sites under purifying selection and/or positive selection within and without disordered regions were performed using WinPepi and SPSS 2.0.

To estimate the molecular evolutionary patterns of FOXP1, FOXP2, FOXP 3, and FOXP4, we applied phylogeny-based maximum likelihood analysis of $\omega$ (non-synonymous/synonymous rate ratio or $\mathrm{dN} / \mathrm{dS}$ ) implemented in the PAML 4.7 package (Yang, 2007). This approach allows the $\omega$ ratio to vary among sites while considering several different codon substitution models. A value of $\omega<1$ indicates potential negative selection, while $\omega=1$ indicates neutrality, and $\omega>1$ indicates positive selection. For the NsSites codon substitution model, likelihood ratio tests (LRT) were performed between neutral models (M1a, nearly neutral, M8a, Beta and $\omega=1$ ) and models that allow positive selection and/or relaxation of functional constraints (M2a, positive selection and M8, Beta + Selection). Using log values from models M1a, M2a, M8a, and M8, we applied an LRT using HyPhy 2.2.0.

The Branch Site Model was also used to detect if different linear motif composition and disorder scores are reflected in different evolutionary rates among Tetrapoda. The phylogeny was a priori divided into two clades, and a LRT was used to evaluate divergences in selective pressures between them, as indicated by different $\omega$ ratios. We employed the clade model type D that assumes two site classes, which was compared with the neutral model M1a by an LRT with two degrees of freedom.

A Bayes empirical Bayes (BEB) approach was considered using CODEML in PAML 4.7 to verify which sites could be under neutral, purifying, or positive selection. The phylogenetic trees used to construct the PAML 4.7 input files were revised as described previously (Meredith et al., 2011; Perelman et al., 2011; Song et al., 2012).

\section{Results and Discussion}

\section{FOXP1, FOXP2, FOXP3 and FOXP4 structures and their intrinsic protein disorder content}

Our analyses revealed that the three paralogous proteins with similar functions and tissue expression, FOXP1, FOXP2, and FOXP4, had high and similar disorder contents $(\sim 70 \%)$. In contrast, FOXP3, which plays a role in immune system regulation, presented a lower disorder degree $(\sim 30 \%)$ relative to its paralogs (Tables 1, S2-S5), according to PONDR-FIT. The patterns of the disordered and ordered
Table 1 - Mean disorder proportion for FOXP proteins by class ${ }^{1}$.

\begin{tabular}{lllll}
\hline Class & FOXP2 & FOXP4 & FOXP1 & FOXP3 $^{2}$ \\
\hline Mammals & 0.7011 & 0.7321 & 0.6915 & 0,3065 \\
Birds & 0.7039 & 0.6858 & 0.6782 & \\
Reptiles & 0.6984 & 0.6827 & 0.6713 & \\
Amphibians & 0.6305 & 0.7068 & NA & \\
\hline
\end{tabular}

${ }^{1}$ Mammals showed significant higher proportions than the other groups, as assessed by the Kruskal-Wallis test, for FOXP1and FOXP4 $(P<0.001)$. Additionally, according to the same test, amphibians presented a lower degree of disorder for FOXP2 $(P<0.01)$.

${ }^{2}$ Only mammalian genes were used for the FOXP3 analysis.

NA: Not available. Since several base pairs in Xenopus laevis FOXP1 sequence are missing, we excluded it from the analysis.

regions, as well as the disorder proportion of orthologous proteins, are relatively conserved among taxonomic groups (Tables 1, S2-S5). However, mammals presented a higher degree of protein disorder than all other organisms for FOXP1 and FOXP4 $(P<0.001$, Table 1). Particularly, amphibians presented a lower degree of disorder for FOXP2 $(\sim 64 \%$, Tables 1 and S3.1) than the other classes $(P<0.01$, Table 1). These FOXP disorder prediction values are, in general, higher than those obtained by other authors (Andersen et al., 2012), but they used just partial proteins and fewer species. Importantly, it is worthy of note that the larger mammalian sample compared to non-mammals may have contributed to these statistical differences in the protein disorder content analysis.

Interestingly, our data reveals that mammals present significantly higher FOXP1 and FOXP4 disorder degrees than the other groups. This finding may be associated with the more complex interaction networks present in mammals, as already proposed for other genetic systems (Disfani et al., 2012), and to a positive correlation between the number of binding partners and disorder scores (Dunker et $a l ., 2000)$. Thus, it is reasonable to speculate that mammalian FOXP1 and FOXP4 present a larger number of binding partners than the other orthologues investigated here.

\section{FOXP1, FOXP2, FOXP3, and FOXP4 and their interaction sites}

Usually, intrinsically disordered proteins are enriched with SLIMs, which play crucial roles in their interaction with other proteins (Tables S6.1-6.4). Here we will briefly describe some selected representative results of the SLIMs compositional analysis. For FOXP1, some of our findings include a Polo-like kinase 1 (PLK) phosphorylation site at position 33 (MOD_PLK), which differentiates Sauropsida (reptiles and birds) from mammals (Table 2). PLK is involved in cell cycle events (Nakajima et al., 2003; Murakami et al., 2010), suggesting some differences in the FOXP1 phosphorylation pattern during the cell cycle between mammals and Sauropsida. 


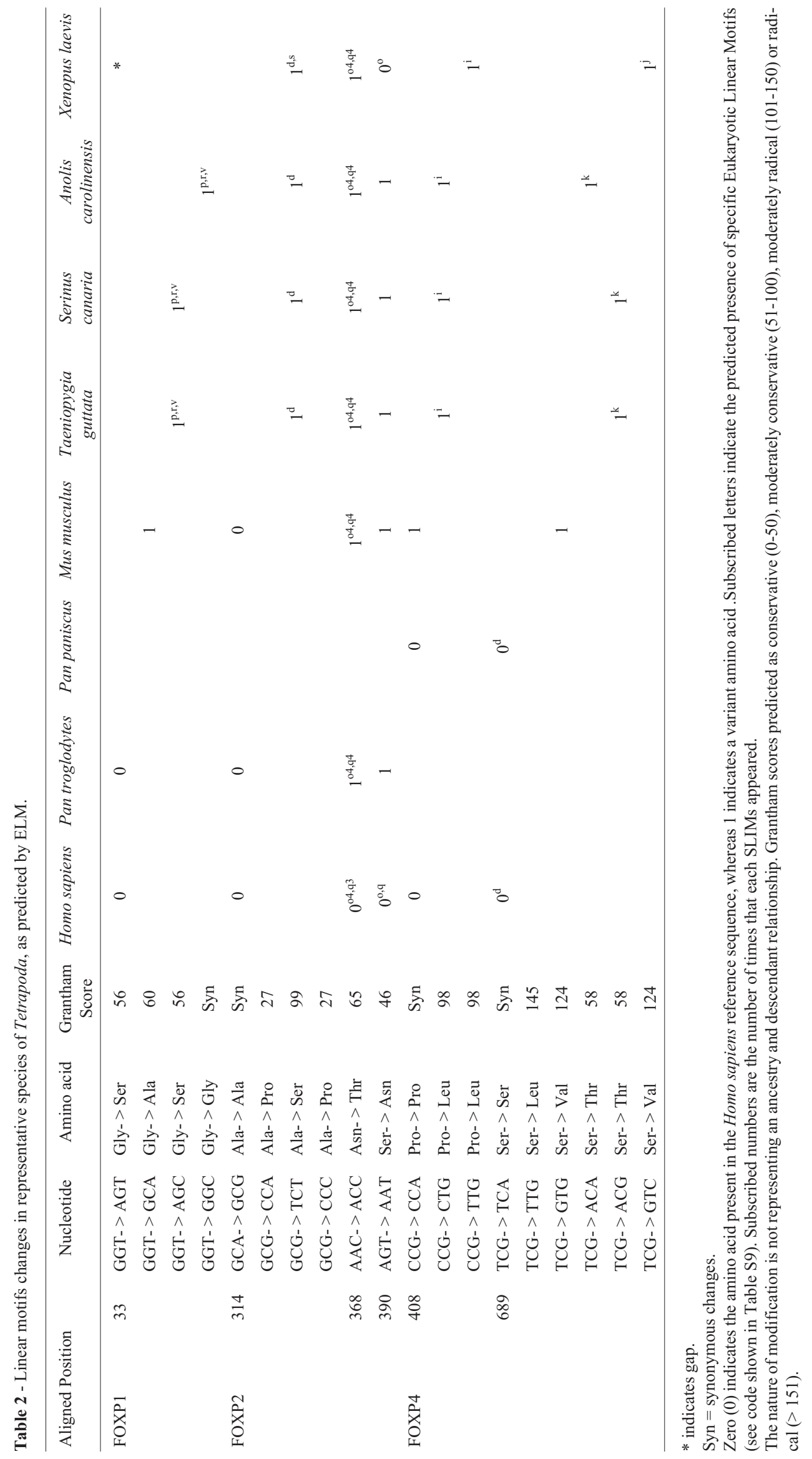


In the case of FOXP2 (Table 2), mammals have lost one DOC_USP7_1 at position 314, which interacts with the deubiquitinating enzyme USP7/HAUSP (herpes virusassociated ubiquitin-specific protease) present in all non-mammals, due to a serine to alanine change. Previous studies have demonstrated that the interaction of USP7 with FOX members regulates oxidative stress responses through ubiquitination (van der Horst et al., 2006). Thus, the possible loss of DOC_USP7_1 in mammals could have a functional implication related to response to oxidative stress.

Two known non-synonymous substitutions between humans (Homo sapiens and Neanderthals) and chimpanzees (FOXP2 Asn325Ser and Thr303Asn) deserve additional attention, since they were related to human speech (Enard et al., 2002, Krause et al., 2007). One of them (Asn325Ser) promotes the gain of two motifs, MOD_CK1_1 and MOD_GSK3_1, in humans due to the presence of a serine at aligned position 390 (Table 2). Both motifs are promoters of phosphorylation by kinases. Interestingly, carnivores also have a serine at this FOXP2 ortholog position (Zhang et al., 2002), leading to a convergence event of the emergence of both MOD_CK1_1 and MOD GSK3 1 motifs observed in humans. Cooper (2006) suggested that phosphorylation by kinase $\mathrm{C}$ in this FOXP2 region may be related to human behavioral traits such as language. However, the other Homo-specific substitution at aligned position 368 (Thr303Asn) led to the loss of a phosphorylation site. Changes in phosphorylation patterns can modulate the regulation of transcription factors and their binding affinity to co-activators and DNA. These changes can in turn alter gene expression, cell growth, and differentiation (Iakoucheva et al., 2004). Thus, our results have one very relevant implication: the loss of this phosphorylation site at position 368/303 can have been as important as the gain of the phosphorylation site at position $390 / 325$ for the evolution of human speech. The phenotype implication of the presence of these SLIMS in carnivores is unknown.

For FOXP3, which was only investigated in mammals (see Material and Methods section), a CK1 phosphorylation site (MOD_CK1_1) is predicted at position 194 (Table 3) for several mammal species, except New World (NW) monkeys (Saimiri boliviensis and Callithrix jacchus) and Tarsius syrichta. Interestingly, these primates present four other linear motifs in this region: MOD_GSK3 1, MOD_ProDkin_1, DEG_SCF_FBW7 1, and DOC_WW_Pin1_4. Therefore, we identified the presence of the same SLIMs in two distinct branches of primates (New World monkeys and Tarsiidae) that live in somewhat similar rainforest environments. As mentioned before, FOXP3 is the only FOXP member playing a role in the immune system, suggesting that at least one of these motifs is associated with the immune response, indicating adaptation through convergence or the maintenance of a primate ancestral state.

Another interesting finding is the sharing of the linear motif LIG_PTAP_UEV_1 between Neanderthals and modern humans due to the Gly175Ser (human position) mutation (Table 4). It has also been suggested that linear motifs mediate interactions between viruses with their hosts (Hagai et al., 2014). In fact, LIG PTAP UEV 1 mediates the binding of several cellular and viral proteins to the UEV domain of the class E vacuolar sorting protein Tsg101 (Göttlinger et al., 1991), and it is essential for the efficient egress of viral particles from many enveloped RNA viruses (Bieniasz, 2006). Our results indicate that this motif may have played an important role in Homo self-immune defense during the Pleistocene.

Regarding FOXP4, a striking difference between mammals and Sauropsida was also found (birds and reptiles, Table 2). For instance, the loss of LIG_CtBP_PxDLS_1 in mammals is due to the substitution of a leucine for proline at aligned position 408, probably after the divergence of Synapsida and Sauropsida. Mendoza et al. (2015) showed that the presence of the CtBP binding region in the bird Taeniopygia guttata has been associated with the potential FOXP4 regulation capacity. This finding for CtBP interaction may be associated with an enhanced potential for transcriptional repression of FOXP4, known for FOXP1 and FOXP2 (Mendoza et al., 2015). At aligned position 689, almost all non-mammals present a motif that interacts with FHA (LIG_FHA_1 or LIG_FHA_2), while mammals present a DOC_USP7_1 motif.

To better understand the role of SLIMs in evolution, we additionally compared members within the FOXP family to verify the number of unique linear motifs in each paralog (Table 5). The number of predicted types of SLIMs range from 28 (FOXP2) to 39 (FOXP4). Furthermore, FOXP3 presents three unique motifs (DOC_PP2B_1, TRG_NLS_MonoCore_2, and TRG_NLS_MonoExtN_4), FOXP1 presents four (DEG_SCF_FBW7_2, LIG_PCNA_PIPBox_1, LIG_WD40_WDR $5 \_1$, and TRG_NES_CRM1_1), while FOXP2 presents no unique SLIM. FOXP4 presents six motifs, among which three (DOC_PP1_RVXF_1, LIG_BRCT_BRAC1_2, and TRG_NLS_MonoExtC_3) are common to almost all species investigated in the current study.

\section{Molecular evolutionary patterns}

Evolutionary tests for FOXP1, FOXP2, FOXP3 and FOXP4 considering all the tetrapod species investigated in this study indicated that the best log-likelihood model is M1a, which assumes purifying selection and neutral $\omega$ values. FOXP 1, FOXP 2 and FOXP 4 present more than $95 \%$ of the sites, with $\omega=0.03066,0.01965$ and 0.02778 , respectively (Table S7), indicating a strong role for purifying selection. FOXP 3 presents $10 \%$ of $\omega$ values equal to 1 , which 
Table 3 - FOXP3 Linear motifs changes in Mammals, as predicted by ELM.

\begin{tabular}{|c|c|c|c|c|c|c|c|}
\hline Aligned Position & & & & 194 & & & \\
\hline Nucleotide & GTG- $>$ ATG & GTG- > ACA & GTG- > TTG & GTG- > GGG & GTG- > GCA & GTG- > GCG & GTG- > ACG \\
\hline Amino acid & Val- $>$ Met & Val- $>$ Thr & Val- > Leu & Val- > Gly & Val- > Ala & Val- > Ala & Val- > Thr \\
\hline Grantham Score & 21 & 69 & 32 & 109 & 64 & 64 & 69 \\
\hline Homo sapiens & $0^{\circ}$ & & & & & & \\
\hline Pan troglodytes & $0^{\circ}$ & & & & & & \\
\hline Pan paniscus & $0^{\circ}$ & & & & & & \\
\hline Gorilla gorilla & $0^{\circ}$ & & & & & & \\
\hline Pongo abellii & $0^{\circ}$ & & & & & & \\
\hline Pongo pygameus & $0^{\circ}$ & & & & & & \\
\hline Hylobates lar & $0^{\circ}$ & & & & & & \\
\hline Nomascus & $0^{\circ}$ & & & & & & \\
\hline Macaca mulatta & $1^{\mathrm{o}}$ & & & & & & \\
\hline Papio anubis & $1^{\mathrm{o}}$ & & & & & & \\
\hline Chlorocebus sabaeus & $1^{\circ}$ & & & & & & \\
\hline Saimiri boliviensis & & $1^{\text {be o } 2 \text { w }}$ & & & & & \\
\hline Callithrix jacchus & & $1^{\text {beoq2w }}$ & & & & & \\
\hline Galeopterus variegatus & $0^{\circ}$ & & & & & & \\
\hline Tarsius syrichta & & $1^{\text {be oq } 2 \mathrm{w}}$ & & & & & \\
\hline Tupaia chinensis & & & $1^{\mathrm{m} 3 \mathrm{jo}}$ & & & & \\
\hline Sorex araneus & & & & $1^{\circ}$ & & & \\
\hline Mus musculus & & & & & $1^{\mathrm{c}}$ & & \\
\hline Cricetulus griseus & & & & & $1^{\mathrm{c}}$ & & \\
\hline Rattus norvegicus & & & & & $1^{\mathrm{c}}$ & & \\
\hline Octodon degus & $1^{\mathrm{o}}$ & & & & & & \\
\hline Oryctolagus cuniculus & & & & & & $1^{\mathrm{o}}$ & \\
\hline Ochotona princeps & & & & & & $1^{\mathrm{o}}$ & \\
\hline Physeter catodon & $1^{\circ}$ & & & & & & \\
\hline Orcinus orca & $1^{\mathrm{o}}$ & & & & & & \\
\hline Camelus ferus & $0^{\circ}$ & & & & & & \\
\hline Bos taurus & $0^{\circ}$ & & & & & & \\
\hline Equus caballus & $1^{\mathrm{o}}$ & & & & & & \\
\hline Ailuropoda melanoleuca & $0^{\circ}$ & & & & & & \\
\hline Felis catus & $0^{\circ}$ & & & & & & \\
\hline Canis lupus familiaris & 0 & & & & & & \\
\hline Vicugna pacos & $0^{\circ}$ & & & & & & \\
\hline Panthera tigris & $0^{\circ}$ & & & & & & \\
\hline Mustela putorius furo & $0^{\circ}$ & & & & & & \\
\hline Odobenus rosmarus & $0^{\circ}$ & & & & & & \\
\hline Leptonychotes weddellii & $0^{\circ}$ & & & & & & \\
\hline Ceratotherium simum & & & & & & & $1^{\text {b e o } 2 \text { w }}$ \\
\hline Eptesicus fuscus & $0^{\circ}$ & & & & & & \\
\hline Myotis brandtii & $0^{\circ}$ & & & & & & \\
\hline Pteropus alecto & $1^{\mathrm{s}}$ & & & & & & \\
\hline Condylura cristata & & & & & & $1^{\circ}$ & \\
\hline Chrysochloris asiatica & $1^{\mathrm{c}}$ & & & & & & \\
\hline Erinaceus europaeus & 1 & & & & & & \\
\hline Echinops telfairi & & & & & & & $1^{\text {be oq2 w }}$ \\
\hline Orycteropus afer afer & & & & & & & $1^{\text {be oq2 w }}$ \\
\hline Loxodonta africana & $1^{\mathrm{ftv}}$ & & & & & & \\
\hline Trichechus manatus & $1^{\mathrm{ew}}$ & & & & & & \\
\hline Dasypus novemcinctus & & & & & & $1^{02 \mathrm{~s}}$ & \\
\hline
\end{tabular}

* indicates gap. Syn = synonymous change. Zero (0) indicates the amino acid present in the Homo sapiens reference sequence whereas 1 indicates a variant amino acid .Subscribed letters indicate the predicted presence of specific Eukaryotic Linear Motifs (see code shown in Table S9). The nature of modification is not representing an ancestry and descendant relationship. Grantham scores predicted as conservative (0-50) moderately conservative (51-100) moderately radical (101-150) or radical (> 151). 
Table 4 - FOXP3-specific changes in primates.

\begin{tabular}{|c|c|c|c|c|}
\hline Organisms & Aligned position & Human position & AA Change & Motifs $^{1}$ \\
\hline \multirow[t]{2}{*}{ Neanderthal and Humans } & 140 & 132 & Pro- $>$ Thr & $(+2)$ DEG_SCF_FBW7_1 \\
\hline & 183 & 175 & Gly- $>$ Ser & (+) LIG_PTAP_UEV_1 \\
\hline Neanderthal & 192 & 184 & Ser- $>$ Leu & (-) MOD_CK1_1, (+) DOC_MAPK_1 \\
\hline Catarrhini & 278 & 270 & Pro- $>$ Ser & $(+)$ MOD_GSK3_1 \\
\hline \multirow[t]{9}{*}{ Haplorhini $^{2}$} & 82 & 74 & Val- > Leu & (-) DOC_WW_Pin1_4, (-)MOD_ProDKin_1 \\
\hline & 97 & 89 & Ser- $>$ Leu & \\
\hline & 129 & 121 & Arg- $>$ His & \\
\hline & 132 & 124 & Asp- $>$ Glu & \\
\hline & 181 & 173 & Ser- $>$ Asn & (-)DOC_WW_Pin1_4, (-) MOD_ProDKin_1 \\
\hline & 246 & 238 & Val- $>$ Met & \\
\hline & 262 & 254 & Gly- > Ser & \\
\hline & 338 & 325 & Phe- > Leu & \\
\hline & 424 & 411 & Phe- > Leu & \\
\hline
\end{tabular}

${ }^{1}+$ : change causes motif gain; -: change causes motif loss.

${ }^{2}$ Excluding Tarsius syrichta.

Table 5 - Number of shared and unique short linear motifs (SLIMs) among Tetrapoda FOXPs.

\begin{tabular}{lcccccc}
\hline Protein & $\begin{array}{c}\text { Total type of } \\
\text { SLIMs }\end{array}$ & $\begin{array}{c}\text { Number of unique } \\
\text { SLIMs }\end{array}$ & $\begin{array}{c}\text { Total SLIMs in } \\
\text { Homo sapiens }\end{array}$ & $\begin{array}{c}\text { Total SLIMs in Pan } \\
s p .\end{array}$ & $\begin{array}{c}\text { Total SLMIs in } \\
\text { Serinus canaria }^{1}\end{array}$ & $\begin{array}{c}\text { Total of species } \\
\text { compared }\end{array}$ \\
\hline FOXP1 & 34 & $4^{2}$ & 132 & 132 & 135 & 50 \\
FOXP2 & 28 & 0 & 143 & 142 & 60 & 54 \\
FOXP3 & 32 & $3^{3}$ & 69 & 62 & -50 & 65 \\
FOXP4 & 39 & $6^{4}$ & 142 & 143 & 160 & 65 \\
\hline
\end{tabular}

${ }^{1}$ Bird, representing Sauropsida.

${ }^{2}$ DEG_SCF_FBW7_2, LIG_PCNA_PIPBox_1, LIG_WD40_WDR5_1, and TRG_NES_CRM1_1);

${ }^{3}$ DOC_PP2B_1, TRG_NLS_MonoCore_2, and TRG_NLS_MonoExtN_4;

${ }^{4}$ FOXP4 presents six motifs, among which three (DOC_PP1_RVXF_1, LIG_BRCT_BRAC1_2, and TRG_NLS_MonoExtC_3) are common to almost all species investigated in the current study.

indicates molecular neutral evolution and/or relaxation of functional constraints.

Additionally, we used the results from the Bayes Empirical Bayes (BEB) test to calculate the posterior probabilities that each codon is under positive selection (Yang, 2007). The BEB values are only significant for M2 and M8 (that include such selection), therefore this last strategy was only adopted to detect eventual functional sites. Such analysis showed four sites in mammals with $\omega>1$ and probability $>91 \%$, but the $p$ value was not significant (Table S7). Regardless, it is important to highlight that one of the sites inferred with $\omega=1.06$ (probability $=98.9 \%$ ) is located at position 194 of FOXP3 (Table 3). This position presents differences in SLIM prediction (MOD_GSK3_1, MOD_ProDkin_1, MOD_CK1_1, DEG_SCF_FBW7_1, and DOC_WW_Pin1_4) in Saimiri boliviensis, Callithrix jacchus, and Tarsius syrichta when compared with the other species. Saimiri boliviensis and Callithrix jacchus probably share the same linear motifs because of their clear and relatively recent common origin, but Tarsius syrichta, which is phylogenetically more distant, may present them because of convergent evolution (Tables S6.3 and 3). MOD_ProdKin_1 is a post-translational modification site phosphorylated by a MAP kinase, while DEG_SCF_FBW7_1 is a degradation site mediated by an important protein complex (Skp, Cullin, F-box containing complex or SCF) that plays a role in checkpoints during the cell cycle (Nguyen Ba et al., 2012). DOC_WW_Pin1_4 interacts with the enzyme Pin1, whose function is also associated with the cell cycle, among others. Additionally, Pin1 regulates the immune response (Gavva et al., 1997; Wulf et al., 2002; Wijchers et al., 2006; Saxena et al., 2010), which 
is a known function of FOXP3. Again, as we identified the presence of the same SLIMs in two distinct primate branches that live in similar environments (rainforest), this allow us to infer that a simple neutral model is insufficient to explain this scenario.

In the case of FOXP4 (Table S8), the Branch Site model indicated that mammals have a $\omega$ value 3.7 times higher than non-mammals (0.66102 versus 0.18012$)$, a result compatible with relaxation of evolutionary pressures. This striking difference $(p<0.001)$ may be attributed to certain changes, such as the absence of the interaction site for CtBP (LIG_CtBP_PxDLS_1) in all mammals (except Sus scrofa). Another structural/functional change that can explain the distinct $\omega$ values observed between mammals and non-mammals is the presence of a glutamine-rich region in mammalian FOXP4, associated to its repression ability.

\section{Conclusion}

Our study reveals some important general and more specific findings. For instance, $70 \%$ of the disorder content has been retained in FOXP1, FOXP2, and FOXP4 orthologs. Some of the results obtained can be associated with taxa-specific conditions, while others may represent molecular convergence. In fact, we found changes at FOXP3 sites with possible functional implications in the primate branch, including the genus Homo. Finally, the FOXP1 and FOXP4 results show instigating differences between mammals and non-mammals, suggesting their role in the emergence of adaptive novelty within the taxon Tetrapoda. Our results indicate that part of the FOXP evolutionary "stability" over a long evolutionary period may be attributed to the maintenance of a similar proportion of disordered regions, but not to amino acid content or linear motifs. Moreover, some of the changes can be interpreted as indicating taxa-specific adaptations, since they are probably functional.

\section{Acknowledgments}

This research was financially supported by the Coordenação de Aperfeiçoamento de Pessoal de Nível Superior (CAPES), and Conselho Nacional de Desenvolvimento Científico e Tecnológico (CNPq).

\section{References}

Akbar AN, Vukmanovic-Stejic M, Taams LS and Macallan DC (2007) The dynamic co-evolution of memory and regulatory CD4+ T cells in the periphery. Nat Rev Immunol 7:231-237.

Andersen KG, Nissen JK and Betz AG (2012) Comparative genomics reveals key gain-of-function events in Foxp3 during regulatory $\mathrm{T}$ cell evolution. Front Immunol 3:1-16.

Benayoun BA, Caburet S and Veitia RA (2011) Forkhead transcription factors: Key players in health and disease. Trends Genet 27:224-232.
Bieniasz PD (2006). Late budding domains and host proteins in enveloped virus release. Virology 344:55-63.

Bowers JM and Konopka G (2012) The role of the FOXP family of transcription factors in ASD. Dis Markers 33:251-260.

Castellano S, Parra G, Sánchez-Quinto FA, Racimo F, Kuhlwilm M, Kircher M, Sawyer S, Fu Q, Heinze A, Nickel B, et al. (2014) Patterns of coding variation in the complete exomes of three Neandertals. Proc Natl Acad Sci U S A 111:66666671.

Cesario JM, Almaidhan AA and Jeong J (2016) Expression of forkhead box transcription factor genes Foxp1 and Foxp2 during jaw development. Gene Expr Patterns 20:111-119.

Coffer PJ and Burgering BMT (2004) Forkhead-box transcription factors and their role in the immune system. Nat Rev Immunol 4:889-899.

Cooper DL (2006) Broca's arrow: Evolution, prediction, and language in the brain. Anat Rec B New Anat 289:9-24.

Dinkel H, Michael S, Weatheritt RJ, Davey NE, Roey KV, Altenberg B, Milchevskaya V, Schneider M, Kühn H, Behrendt A, et al. (2012). ELM - The database of eukaryotic linear motifs. Nucleic Acids Res 40:1-10.

Dinkel H, Michael S, Weatheritt RJ, Davey NE, Van Roey K, Altenberg B, Toedt G, Uyar B, Seiler M, Budd A, et al. (2014) The eukaryotic linear motif resource ELM: 10 years and counting. Nucleic Acids Res 42:1-8.

Disfani FM, Hsu WL, Mizianty MJ, Oldfield CJ, Xue B, Dunker AK, Uversky VN and Kurgan L (2012) MoRFpred, a computational tool for sequence-based prediction and characterization of short disorder-to-order transitioning binding regions in proteins. Bioinformatics 28:75-83

Dunker AK, Obradovic Z, Romero P, Garner EC and Brown CJ (2000) Intrinsic protein disorder in complete genomes. Genome Inform Ser Workshop Genome Inform 11:161-171.

Enard W, Przeworski M, Fisher SE, Lai CS, Wiebe V, Kitano T, Monaco AP and Pääbo S (2002) Molecular evolution of FOXP2, a gene involved in speech and language. Nature 418:869-872.

Enard W (2011) FOXP2 and the role of cortico-basal ganglia circuits in speech and language evolution. Curr Opin Neurobiol 21:415-424

Forman-Kay JD and Mittag T (2013) From sequence and forces to structure, function, and evolution of intrinsically disordered proteins. Structure 21:1492-1499.

Gavva NR, Gavva R, Ermekova K, Sudol M and Shen C-K (1997) Interaction of WW domains with hematopoietic transcription factor $\mathrm{p} 45 / \mathrm{NF}-\mathrm{E} 2$ and RNA polymerase II. J Biol Chem 272:24105-24109.

Göttlinger HG, Dorfman T, Sodroski JG and Haseltine WA (1991) Effect of mutations affecting the p6 gag protein on human immunodeficiency virus particle release. Proc Natl Acad Sci U S A 88:3195-3199.

Gould CM, Diella F, Via A, Puntervoll P, Gemünd C, ChabanisDavidson S, Michael S, Sayadi A, Bryne JC, Chica C, et al. (2010) ELM: The status of the 2010 eukaryotic linear motif resource. Nucleic Acids Res 38:167-180.

Hagai T, Azia A, Babu MM and Andino R (2014) Use of host-like peptide motifs in viral proteins is a prevalent strategy in host-virus interactions. Cell Rep 7:1729-1739.

Huang H and Sarai A (2012) Analysis of the relationships between evolvability, thermodynamics, and the functions of 
intrinsically disordered proteins/regions. Comput Biol Chem 41:51-57.

Iakoucheva LM, Radivojac P, Brown CJ, O'Connor TR, Sikes JG, Obradovic Z and Dunker AK (2004) The importance of intrinsic disorder for protein phosphorylation. Nucleic Acids Res 32:1037-1049.

Krause J, Lalueza-Fox C, Orlando L, Enard W, Green RE, Burbano HA, Hublin JJ, Hänni C, Fortea J, de la Rasilla M, et al. (2007) The derived FOXP2 variant of modern humans was shared with Neandertals. Curr Biol 17:1908-1912.

Lam EW, Brosens JJ, Gomes AR and Koo CY (2013) Forkhead box proteins: Tuning forks for transcriptional harmony. Nat Rev Cancer 13:482-495.

Li G, Wang J, Rossiter SJ, Jones G and Zhang S (2007) Accelerated FOXP2 evolution in echolocating bats. PLoS One 2:e900.

Liu J, Perumal NB, Oldfield CJ, Su EW, Uversky VN and Dunker AK (2006) Intrinsic disorder in transcription factors. Biochemistry 45:6873-6888.

Liu Z and Huang Y (2014) Advantages of proteins being disordered. Protein Sci 23:539-550.

Mendoza E, Tokarev K, Düring DN, Retamosa EC, Weiss M, Arpenik N and Scharff C (2015) Differential co-expression of FOXP1, FOXP2 and FOXP4 in zebra finch (Taeniopygia guttata) song system. J Comp Neurol 523:1318-1340.

Meredith RW, Janecka JE, Gatesy J, Ryder OA, Fisher CA, Teeling EC, Goodbla A, Eizirik E, Simão TL, Stadler T, et al. (2011) Impacts of the Cretaceous terrestrial revolution and $\mathrm{KPg}$ extinction on mammal diversification. Science 334:521-524

Morris G and Fanucchi S (2016) A key evolutionary mutation enhances DNA binding of the FOXP2 forkhead domain. Biochemistry 55:1959-1967.

Murakami H, Aiba H, Nakanishi M and Murakami-Tonami Y (2010) Regulation of yeast forkhead transcription factors and FOXM1 by cyclin-dependent and polo-like kinases. Cell Cycle 9:3233-3242.

Nakajima H, Toyoshima-Morimoto F, Taniguchi E and Nishida E (2003) Identification of a consensus motif for PIK (Polo-like kinase) phosphorylation reveals Myt1 as a Plk1 substrate. J Biol Chem 278:25277-25280.

Neduva V and Russell RB (2005) Linear motifs: Evolutionary interaction switches. FEBS Lett 579:3342-3345.

Nguyen Ba AN, Yeh BJ, van Dyk D, Davidson AR, Andrews BJ, Weiss EL and Moses AM (2012) Proteome-wide discovery of evolutionary conserved sequences in disordered regions. Sci Signal 5:rs1.

Perelman P, Johnson WE, Roos C, Seuánez HN, Horvath JE, Moreira MA, Kessing B, Pontius J, Roelke M, Rumpler Y, et al. (2011) A molecular phylogeny of living primates. PLoS Genet 7:e1001342.

Pigliucci M (2008) Is evolvability evolvable? Nat Rev Genet 9:75-82

Potenza E, Di Domenico T, Walsh I and Tosatto SC (2014) MobiDB 2.0: An improved database of intrinsically disordered and mobile proteins. Nucleic Acids Res 43:D315D320.

Santos ME, Athanasiadis A, Leitão AB, DuPasquier L and Sucena E (2011) Alternative splicing and gene duplication in the evolution of the FOXP gene subfamily. Mol Biol Evol 28:237-247.
Saxena U, Owens L, Graham JR, Cooper GM and Hansen U (2010) Prolyl Isomerase Pin1 regulates Transcription Factor LSF (TFCP2) by facilitating dephosphorylation at twoserine-proline motifs. J Biol Chem 285:31139-31147.

Schön C, Wochnik A, Rössner A, Donow C and Knöchel W (2006) The FOXP subclass in Xenopus laevis development. Dev Genes Evol 216:641-646.

Song S, Liu L, Edwards SV and Wu S (2012) Resolving conflict in eutherian mammal phylogeny using phylogenomics and the multispecies coalescent model. Proc Natl Acad Sci U S A 109:14942-14947.

Song X, Wang Y and Tang Y (2013) Rapid diversification of FOXP2 in teleosts through gene duplication in the teleost-specific whole genome duplication event. PLoS One 8:e83858.

Song X, Tang Y and Wang Y (2016) Genesis of the vertebrate FoxP subfamily member genes occurred during two ancestral whole genome duplication events. Gene 588:156-162

Takahashi K, Liu FC, Oishi T, Mori T, Higo N, Hayashi M, Hirokawa K and Takahashi H (2008) Expression of FOXP2 in the developing monkey forebrain: Comparison with the expression of the genes FOXP1, PBX3, and MEIS2. J Comp Neurol 509:180-189.

van der Horst A, de Vries-Smits AM, Brenkman AB, van Triest $\mathrm{MH}$, van den Broek N, Colland F, Maurice MM and Burgering BM (2006) FOXP4 transcriptional activity is regulated by monoubiquitination and USP7/HAUSP. Nat Cell Biol 8:1064-1073.

Wagner GP and Lynch VJ (2008) The gene regulatory logic of transcription factor evolution. Trends Ecol Evol 23:377385.

Wijchers PJEC, Burbach JPH and Smidt MP (2006) In control of biology: Of mice, man and foxes. Biochem J 397:233-246.

Wulf G, Liou Y and Ryo A (2002) Role of Pin1 in the regulation of p53 stability and p21 transactivation, and cell cycle checkpoints in response to DNA damage. J Biol Chem 277:47976-47979.

Xue B, Dunbrack RL, Williams RW, Dunker AK and Uversky VN (2010a) PONDR-FIT: A meta-predictor of intrinsically disordered amino acids. Biochim Biophys Acta 1804:9961010.

Xue B, Williams RW, Oldfield CJ, Dunker AK and Uversky VN (2010b) Archaic chaos: Intrinsically disordered proteins in Archaea. BMC Syst Biol 4:S1.

Xue B, Brown CJ, Dunker AK and Uversky VN (2013) Intrinsically disordered regions of p53 family are highly diversified in evolution. Biochim Biophys Acta 1834:725-738.

Yang Z (2007) PAML 4: Phylogenetic analysis by maximum likelihood. Mol Biol Evol 24:1586-1591.

Zhang J, Webb DM and Podlaha O (2002) Accelerated protein evolution and origins of human-specific features: FOXP2 as an example. Genetics 162:1825-1835.

\section{Internet Resources}

http://cdna.eva.mpg.de/, (last accessed March, 2015).

http://ensembl.org/, (last accessed October, 2014).

http://ncbi.nlm.nih.gov, (last accessed October, 2014).

\section{Supplemental Material}

The following online material is available for this article: 
Table S1. List of Tetrapoda species and the respective sequence codes retrieved for each of the FOXP subfamily members studied.

Table S2.1. Disorder proportion of FOXP1 orthologues. Table S2.2. Ordered regions of FOXP1 orthologues.

Table S3.1. Disorder proportion of FOXP2 orthologues.

Table S3.2. Ordered regions of FOXP2 orthologues.

Table S4.1. Disorder proportion of FOXP3 orthologues. Table S4.2. Ordered regions of FOXP3 orthologues.

Table S5.1. Disorder proportion of FOXP4.

Table S5.2. Ordered regions of FOXP4.

Table S6.1. Whole protein comparison for FOXP1 linear motifs content.
Table S6.2. Whole protein comparison for FOXP2 linear motifs content.

Table S6.3. Whole protein comparison for FOXP3 linear motifs content.

Table S6.4. Whole protein comparison for FOXP4 linear motifs content.

Table S7. Estimated parameters under different codon substitution models for forkhead $\mathrm{P}$ subfamily genes.

Table S8. Branch site model for FOXP genes.

Table S9. Eukaryotic linear motifs and their functions.

Associate Editor: Louis Bernard Klaczko

License information: This is an open-access article distributed under the terms of the Creative Commons Attribution License (type CC-BY), which permits unrestricted use, distribution and reproduction in any medium, provided the original article is properly cited. 